\title{
Metal organic frameworks as a drug delivery system for flurbiprofen
}

This article was published in the following Dove Press journal:

Drug Design, Development and Therapy

II September 2017

Number of times this article has been viewed

\author{
Muder AL Haydar ${ }^{1,2}$ \\ Hussein Rasool Abid Ab, $^{3,4}$ \\ Bruce Sunderland ${ }^{2}$ \\ Shaobin Wang ${ }^{5,6}$
}

'Pharmaceutics Department, College of the Pharmacy, University of Kerbala, Kerbala, Iraq; ${ }^{2}$ Pharmaceutics Department, School of Pharmacy, Faculty of Health Sciences, Curtin University, Perth, WA, Australia;

${ }^{3}$ Department of Chemical Engineering, Curtin University, Perth, WA,

Australia; ${ }^{4}$ College of Applied Medical Sciences, University of Kerbala, Kerbala, Iraq; ${ }^{5}$ School of Pharmacy, Faculty of Health Sciences, Curtin University, Perth, WA, Australia; ${ }^{6}$ Department of Chemical Engineering, School of Chemical and Petroleum Engineering, Faculty of Science and Engineering, Curtin University, Perth, WA, Australia
Correspondence: Muder AL Haydar Pharmacy School, Curtin University, Kent Street, Bentley, Perth, WA 6I02, Australia

Tel +6I 474644535

Email muder.alhaydar@curtin.edu.au
Background: Metal organic frameworks (MOFs) have attracted more attention in the last decade because of a suitable pore size, large surface area, and high pore volume. Developing biocompatible MOFs such as the MIL family as a drug delivery system is possible.

Purpose: Flurbiprofen (FBP), a nonsteroidal anti-inflammatory agent, is practically insoluble in aqueous solution, and, therefore, needs suitable drug delivery systems. Different biocompatible MOFs such as Ca-MOF and Fe-MILs (53, 100, and 101) were synthesized and employed for FBP delivery.

Patients and methods: A sample of $50 \mathrm{mg}$ of each MOF was mixed and stirred for $24 \mathrm{~h}$ with $10 \mathrm{~mL}$ of $5 \mathrm{mg}$ FBP in acetonitrile (40\%) in a sealed container. The supernatant of the mixture after centrifuging was analyzed by high-performance liquid chromatography to determine the loaded quantity of FBP on the MOF. The overnight-dried solid material after centrifuging the mixture was analyzed for loading percent using X-ray diffraction, Fourier-transform infrared spectroscopy, scanning electron microscopy, nuclear magnetic resonance, and FBP release profile.

Results: The loading values of FBP were achieved at $10.0 \% \pm 1 \%, 20 \% \pm 0.8 \%, 37 \% \pm 2.3 \%$, and $46 \% \pm 3.1 \%$ on Ca-MOF, Fe-MIL-53, Fe-MIL-101, and Fe-MIL-100, respectively. The FBP release profiles were investigated in a phosphate buffer solution at $\mathrm{pH}$ 7.4. The total release of the FBP after 2 days was obtained at 72.9, 75.2, 78.3, and 90.3\% for Ca-MOF, Fe-MIL-100, Fe-MIL-53, and Fe-MIL-101, respectively.

Conclusion: The MOFs are shown to be a promising drug delivery option for FBP with a significant loading percent and relatively prolonged drug release.

Keywords: porous MOF, FBP, drug loading, drug release

\section{Introduction}

Over the last 20 years, a number of different nanoparticle-based strategies have been developed to improve the efficacy of conventional drug delivery. ${ }^{1,2}$ Porous and tunable hybrid materials, metal organic frameworks (MOFs), are promising candidates as potential drug carriers, because of their remarkably large surface areas and excessively high porosities. ${ }^{3,4}$ The adjustment of the framework's functional groups and pore size make it advantageous over rigid nanoparticle carriers in biomedical applications. ${ }^{5}$ Although several types of organic carriers at a nanoscale level such as micelles, liposomes, and dendrimers ${ }^{6-9}$ have been employed for drug delivery, the drug release is difficult to control with an absence of tunable porosity. ${ }^{10}$ In contrast, MOF nanoparticles have a high loading capacity and controlled drug release properties. ${ }^{2}$

Moreover, the toxicity and biocompatibility of metals and organic linkers used in MOFs have been evaluated. ${ }^{11}$ Biocompatible metals including $\mathrm{Ca}, \mathrm{Cu}, \mathrm{Mn}, \mathrm{Mg}, \mathrm{Zn}$, and Fe may accumulate in the body during the drug release process. However, Fe-MIL-88A 
is approved as an oral iron supplement. ${ }^{11}$ Toxicological studies on Fe-MIL-88 and Fe-MIL-101 have indicated an optimistic view on the toxicity of MOFs. ${ }^{12,13}$ Organic linkers are either exogenous or endogenous compounds. Exogenous organic linkers are produced from polycarboxylates, imidazolates, pyridyl, and amines. ${ }^{14}$ Several endogenous organic linkers are ingredients of the body composition, and have been used for synthesis of biocompatible amino acid MOFs and nucleobase MOFs. ${ }^{15}$

Currently, the most studied MOFs for drug delivery have used Fe metal with bio-organic linkers, such as MILs $\left(53,88,100\right.$, and 101). ${ }^{11,16,17}$ Bernini et al studied a potential carrier of ibuprofen by comparing validated simulation data for ibuprofen adsorption and release in MIL-53, MIL-100, and MIL-101 with interesting CDMOF-1, MOF-74, and BioMOF-100. ${ }^{18}$ Porous iron carboxylate MOFs have been successfully used for encapsulation of a challenging antitumor molecule performing unprecedented loadings exceeding $25 \mathrm{wt} \%$, which may allow the administration of high doses of drugs using low amounts of MOFs. ${ }^{19,20}$ Bio-application studies of a calcium inorganic linker-based MOF are few, however; Sumida et al explored the potential use of calcium carbonate as a precursor to MOF materials to form highly crystalline products. $^{21}$

Flurbiprofen (FBP) (Figure 1) is a hydrophobic and potent acidic nonsteroidal anti-inflammatory drug, which has a molecular weight of 244.3, pKa of 4.6, and $\log P$-value of 4.16. It is practically water insoluble. ${ }^{22}$ To reduce the potential side effects of FBP, numerous delivery systems have been designed, such as transdermals, microspheres, microsponges, and niosomes. ${ }^{23}$ To maintain a prolonged therapeutic activity, the rate of FBP release in different surfactants is low at $10 \%-60 \%{ }^{24}$ This study aims to develop a substantially improved loading percent and release profile

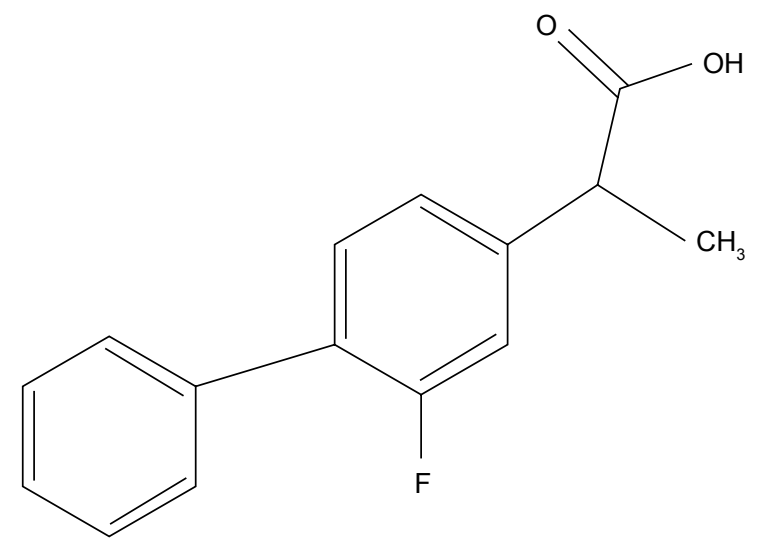

Figure I Flurbiprofen chemical structure. by employing Ca-MOF and different Fe-MILs (MIL-53, MIL-100, and MIL-101).

\section{Materials and methods Materials}

Flurbiprofen was purchased from PCCA Houston, Texas. Terephthalic acid $\left(\mathrm{C}_{8} \mathrm{H}_{6} \mathrm{O}_{4}, 98 \%\right)$, calcium nitrate tetrahydrate $\left(\mathrm{Ca}\left(\mathrm{NO}_{3}\right)_{2} \cdot 4 \mathrm{H}_{2} \mathrm{O}, 99 \%\right)$, iron (III) chloride hexahydrate $\left(\mathrm{FeCl}_{3} \cdot 6 \mathrm{H}_{2} \mathrm{O}, 98 \%\right)$, trimesic acid $\left(\mathrm{C}_{9} \mathrm{H}_{6}, 98 \%\right)$, acetic acid $\left(\mathrm{C}_{2} \mathrm{H}_{4} \mathrm{O}_{2} 100 \%\right)$, dimethylformamide (DMF, $98 \%)$, and ethanol $\left(\mathrm{C}_{2} \mathrm{H}_{6} \mathrm{O},>95 \%\right)$ were purchased from Sigma, Sydney, NSW, Australia and used without further purification. Acetonitrile $\left(\mathrm{C}_{2} \mathrm{H}_{3} \mathrm{~N} 99.9 \%\right)$ and phosphoric acid $\left(\mathrm{H}_{3} \mathrm{PO}_{4} 85 \%\right)$ were supplied by Fisher Chemical, Belgium.

\section{Synthesis of MOFs}

Solvothermal and hydrothermal synthesis procedure was used to prepare the MOFs by Teflon-lined steel autoclave at mild temperature (Teflon-line autoclave [4744 Acid Digestion Bomb] with a capacity of $125 \mathrm{~mL}$ supplied by John Morris Scientific Pty Ltd, Australia).

Ca-MOF was synthesized solvothermally by using a multisolvent solution in a Teflon-lined steel autoclave. A mixture of calcium nitrate tetrahydrate $(18 \mathrm{mmol})$, terephthalic acid (9 mmol), acetic acid (407 mmol), ethanol $48.6 \mathrm{~mL}$, and distilled water $(13.5 \mathrm{~mL})$ was incubated at $165^{\circ} \mathrm{C}$ for 1 day. Then it was immersed in $100 \mathrm{~mL}$ of acetonitrile (ACN) for 4 days until a white powder precipitated. The white powder was filtered by vacuum filtration and dried at $100^{\circ} \mathrm{C}$ and, finally, it was dried at $170^{\circ} \mathrm{C}$ overnight.

Fe-MIL-53 was synthesized specifically by mixing terephthalic acid $(10 \mathrm{mmol})$ with $\mathrm{FeCl}_{3} \cdot 6 \mathrm{H}_{2} \mathrm{O}(10 \mathrm{mmol})$ in $50 \mathrm{~mL}$ of DMF at $150^{\circ} \mathrm{C}$ for $65 \mathrm{~h}$. The product was centrifuged and then washed in water many times. The obtained solid was filtered, washed with DMF, and stirred successively in $200 \mathrm{~mL}$ of methanol for $10 \mathrm{~h}$ and in $200 \mathrm{~mL}$ of water for $15 \mathrm{~h} .{ }^{25}$ After drying in an oven at $70^{\circ} \mathrm{C}$, a yellowish-brown powder was recovered.

Fe-MIL-101 was prepared by mixing terephthalic acid ( $2.5 \mathrm{mmol})$ with $\mathrm{FeCl}_{3} \cdot 6 \mathrm{H}_{2} \mathrm{O}(5 \mathrm{mmol})$ in $30 \mathrm{~mL}$ of DMF. Then the mixture was transferred to an autoclave of $45 \mathrm{~mL}$ and heated in an oven at $110^{\circ} \mathrm{C}$ for $20 \mathrm{~h} .{ }^{26}$ The product was immersed in hot ethanol twice during $3 \mathrm{~h}$. After drying in an oven at $70^{\circ} \mathrm{C}$, an orange-brownish powder was recovered.

Fe-MIL-100 was prepared by mixing of $5 \mathrm{mmol}$ of $\mathrm{FeCl}_{3} \cdot 6 \mathrm{H}_{2} \mathrm{O}$ with $\mathrm{H}_{3} \mathrm{BTC}$ (trimesic acid) in $36 \mathrm{~mL}$ of distilled water inside an autoclave of $125 \mathrm{~mL}$ and heated in an oven at $160^{\circ} \mathrm{C}$ for $15 \mathrm{~h}$. After cooling the autoclave, the suspension 
was centrifuged and the solid was washed successively in hot water $\left(350 \mathrm{~mL}, 70^{\circ} \mathrm{C}\right.$ and $\left.3 \mathrm{~h}\right)$ and in hot ethanol $\left(250 \mathrm{~mL}, 65^{\circ} \mathrm{C}\right.$ and $\left.3 \mathrm{~h}\right)$. The obtained orange-brownish powder was dried at $90^{\circ} \mathrm{C}$ overnight.

\section{Characterization of MOFs}

$\mathrm{X}$-ray powder diffraction (XRPD) patterns were obtained using a D8 Advance (Bruker AXS, Germany), with a copper $\mathrm{K} \alpha$ radiation source $(40 \mathrm{kV}$ and $40 \mathrm{~mA}$ ) with a Lynx Eye detector. The 2-theta scan range was of 5-40 degrees. Fourier-transform infrared (FTIR) spectra of all the MOFs and loaded MOFs were measured using a PerkinElmer FTIR spectrometer in the range of $650-3,800 \mathrm{~cm}^{-1}$. The surface morphology imaging of the MOFs was achieved by using Zeiss Neon 40 EsB field-emission scanning electron microscopy (SEM) with SmartSEM software. Nuclear magnetic resonance (NMR) spectroscopy provides a better understanding of the loading behavior in the drug delivery system. NMR spectra were acquired on a Bruker Avance Ultrashield $400 \mathrm{MHz}$ spectrometer and the NMR spectra were referenced to their solvents: deuterium oxide $\left(\mathrm{D}_{2} \mathrm{O}, 1 \mathrm{H}, \delta 4.79 \mathrm{ppm}\right)$. Also, textural properties of MOFs were characterized by $\mathrm{N}_{2}$ adsorption-desorption isotherms, which were obtained at $-196^{\circ} \mathrm{C}$ by Micromeritics, Tristar instrument, to determine the specific surface area, pore size, and volume.

\section{Incorporation of FBP with MOFs}

The FBP calibration assay was analyzed by high-performance liquid chromatography (HPLC) (Shimadzu 20 AC) at a range of FBP concentrations $(10,20,30,40,50$, and $60 \mu \mathrm{g} / \mathrm{mL}$ in methanol [90\%]) and the mobile phase was $0.1 \mathrm{M}$ phosphate buffer solution (PBS) pH 6.8 and ACN (40\%). A sample of $50 \mathrm{mg}$ of FBP was dissolved in $10 \mathrm{~mL}$ of $\mathrm{ACN}(40 \%)$ to make an FBP solution of $5 \mathrm{mg} / \mathrm{mL}$. An aliquot of $100 \mu \mathrm{L}$ was withdrawn and diluted to $10 \mathrm{~mL}$ with $\mathrm{ACN}(40 \%)$ and analyzed by HPLC to determine the amount of FBP before loading. The MOFs were dried at $120^{\circ} \mathrm{C}$ for $6 \mathrm{~h}$ before loading in the FBP solution. A dried sample of $50 \mathrm{mg}$ of each MOF was separately weighed and mixed with the FBP solution in $20 \mathrm{~mL}$ glass containers. After sealing the container tightly, the mixture was stirred (at $100 \mathrm{rpm}$ ) for $24 \mathrm{~h}$ at room temperature by a magnetic stirrer. The supernatant was collected after centrifugation (4,000 rpm, $15 \mathrm{~min}$ ). The material of MOF with loaded FBP (FBP@MOFs) in the bottom of centrifugal tube was immediately washed with $10 \mathrm{~mL}$ of $40 \% \mathrm{ACN}$ and centrifuged to remove FBP adsorbed on the outer surface of MOFs. Then the FBP@MOF material was dried overnight at $60^{\circ} \mathrm{C}$ in an oven. An aliquot of $100 \mu \mathrm{L}$ was withdrawn from the supernatant, diluted to $10 \mathrm{~mL}$ with ACN (40\%), and analyzed by HPLC to determine the remaining amount of FBP in the solution after loading. The loading amount of FBP can be calculated by subtracting the amount of FBP in the supernatant solution from the amount of FBP before loading. The loading percentage can be calculated by employing the following equation:

$$
\text { Drug loading } \%=\frac{\text { Weight of drug in the MOF }}{\text { Weight of MOF }} \times 100
$$

\section{Release profiles}

A predetermined quantity of FBP@MOF accurately weighed was submerged into $10 \mathrm{~mL}$ of preheated dissolution medium (50 mmol PBS) at $\mathrm{pH} 7.4$ confined in sealed $20 \mathrm{~mL}$ capacity glass vials maintained at $37^{\circ} \mathrm{C} \pm 1^{\circ} \mathrm{C}$ with a constant stirring at a rate of around $75 \mathrm{rpm}$. At predetermined time intervals $(0.25,0.5,1,2,3,5,8,12,24$, and $48 \mathrm{~h})$, an aliquot of $50 \mu \mathrm{L}$ was withdrawn and replaced with the same volume of fresh dissolution medium. The aliquots were diluted 100 times with the same buffer solution and filtered by $0.2 \mu \mathrm{m}$ syringe filter and analyzed using HPLC. A correction of the FBP amount in dissolution medium extracted was calculated regarding the FBP lost in each aliquot. The released percent of FBP was calculated according to the following equation:

$$
\begin{aligned}
& \% \text { Release } \\
& =\frac{\text { Actual FBP released at any time }(\mathrm{mg})}{\text { Amount of FBP loaded within MOF }(\mathrm{mg})} \times 100
\end{aligned}
$$

\section{Results}

\section{Characterization of MOFs}

XRPD data indicated that the crystallinity of FBP@MOFs was significantly changed in respect to the unloaded MOFs (Figure 2). The level and intensity of the peaks were significantly reduced. The peak positions in the XRPD patterns were similar for the FBP@MOF and the unloaded MOF. This indicates that the MOF structures were stable after FBP loading within all MOFs.

FTIR spectra of pure MOFs and FBP@MOFs are shown in Figure 3. The peak at 1,694 was assigned to the carboxyl group in FBP. The peak at $1,690 \mathrm{~cm}^{-1}$ in Fe-MIL-53, Fe-MIL100, and Fe-MIL-101 and FBP-loaded samples was assigned to $\mathrm{C}=\mathrm{O}$ stretching. The peak at $1,621 \mathrm{~cm}^{-1}$ in $\mathrm{Ca}-\mathrm{MOF}$ and FBP@Ca-MOF was also assigned to $\mathrm{C}=\mathrm{O}$ stretching. The shift in carboxyl group from 1,694 to $1,621 \mathrm{~cm}^{-1}$ is because of employing two acids (terephthalic acid and acetic acid) in the 

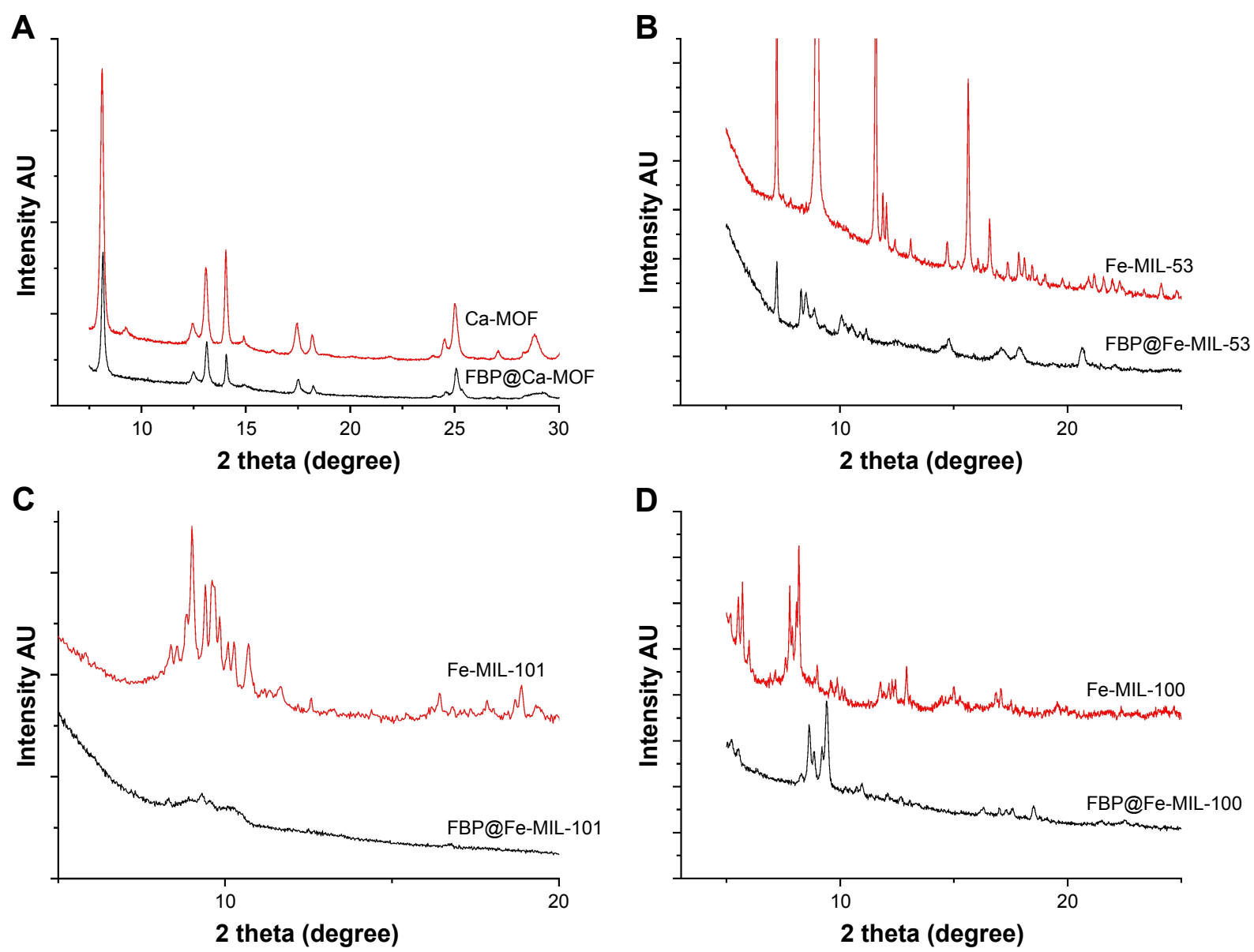

Figure 2 X-ray powder diffraction of (A) Ca-MOF and FBP@Ca-MOF, (B) Fe-MIL-53 and FBP@Fe-MIL-53, (C) Fe-MIL-I0I and FBP@Fe-MIL-I0I, (D) Fe-MIL-I00 and FBP@Fe-MIL-100.

Abbreviations: FBP, flurbiprofen; MOF, metal organic framework.

preparation of the Ca-MOF; the larger shift of the carboxyl group is due to the stronger acidity, ${ }^{27}$ while one acid type was used with the other MOFs. The FTIR spectrum shows the stretching of the carboxyl group of all loaded MOFs indicating the presence of FBP molecules in all FBP@MOFs.

SEM images of MOFs and FBP-loaded MOFs, as shown in Figure 4, are represented by $\mathrm{A}, \overline{\mathrm{A}}, \mathrm{B}, \overline{\mathrm{B}}, \mathrm{C}, \overline{\mathrm{C}}, \mathrm{D}$, and $\overline{\mathrm{D}}$, which are Ca-MOF, FBP@Ca-MOF, Fe-MIL-53, FBP@ Fe-MIL-53, Fe-MIL-101, FBP@Fe-MIL-101, Fe-MIL-100, and FBP@Fe-MIL-100, respectively. Figure 4 shows the nature of microcrystallinity and relatively similar shape and size of each raw MOF and the corresponding loaded one. However, the nature of the surface morphology of FBP@, MOFs was changed. Ca-MOF and the corresponding loaded one show that they are rod-shaped, while Fe-MIL-53 and the corresponding loaded one show a platelet shape with an average length of crystals in the micron range. Fe-MIL-100 and the corresponding loaded one have a triangular base pyramid shape, while Fe-MIL-101 and the corresponding loaded one have a needle shape. The SEM images of FBP@, MOFs indicate an inflated morphology as compared to the bare MOF, suggesting a filling of the drug in the porous MOF. This result indicates loading of FBP within the MOFs.

NMR spectroscopy was employed for investigation of FBP loading within all MOFs. The presence of a signal at $1.25 \mathrm{ppm}$ of pure FBP and its loaded MOFs (Figure S1) was an indication of resonance spectroscopy of methyl group in the FBP structure (Figure 1). This result indicates the presence of FBP molecules within all MOFs.

$\mathrm{N}_{2}$ adsorption-desorption isotherms were obtained and analyzed to determine the surface area, pore size, and pore volume of each MOF. Based on the $\mathrm{N}_{2}$ adsorption isotherms, the Brunauer-Emmett-Teller (BET) surface area of all MOFs is reported in Table 1. The pore volumes obtained from the isotherm at $\mathrm{P} / \mathrm{Po}=1$ (Figure $\mathrm{S} 2$ ) and the average pore sizes of all MOFs are also listed in Table 1. Figure S2 shows the degree of hysteresis and mesopore content in $\mathrm{Ca}-\mathrm{MOF}$, Fe-MIL-53, Fe-MIL-100, and Fe-MIL-101, respectively. 

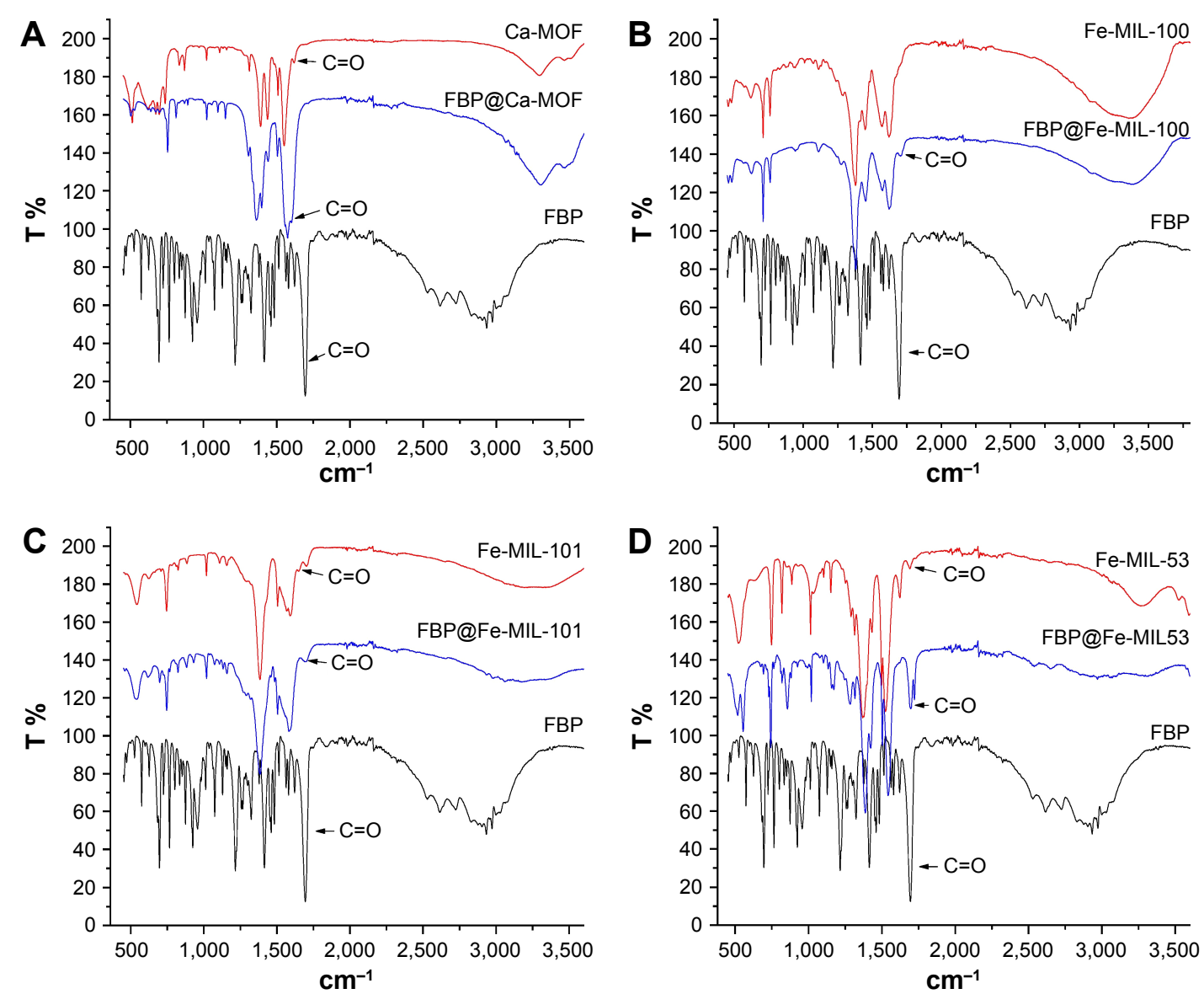

Figure 3 Fourier-transform infrared spectra of (A) Ca-MOF (red) and FBP@Ca-MOF (blue), (B) Fe-MIL-I00 (red) and FBP@Fe-MIL-I00 (blue), (C) Fe-MIL-I0I (red) and FBP@Fe-MIL-I0I (blue) and (D) Fe-MIL-53 (red) and FBP@Fe-MIL-53 (blue).

Note: FBP spectrum (black).

Abbreviations: FBP, flurbiprofen; MOF, metal organic framework.

The sharp increase in $\mathrm{N}_{2}$ adsorption at a pressure close to 1 demonstrates the availability of macropore in their structure as shown clearly with a wide range in the isotherms of $\mathrm{Ca}$ MOF, Fe-MIL-53, and Fe-MIL-101, which was very limited in the isotherm of Fe-MIL-100. Also, a wide hysteresis via a long range of relative pressure in $\mathrm{Ca}-\mathrm{MOF}$ followed by $\mathrm{Fe}-$ MIL-53 and Fe-MIL-101 represents the mesopore content due to ink-bottle pore effect. ${ }^{28}$ However, the sharp increase in $\mathrm{N}_{2}$ adsorption at a low relative pressure represents the micropore, which is supported by overlapping $\mathrm{N}_{2}$ adsorption-
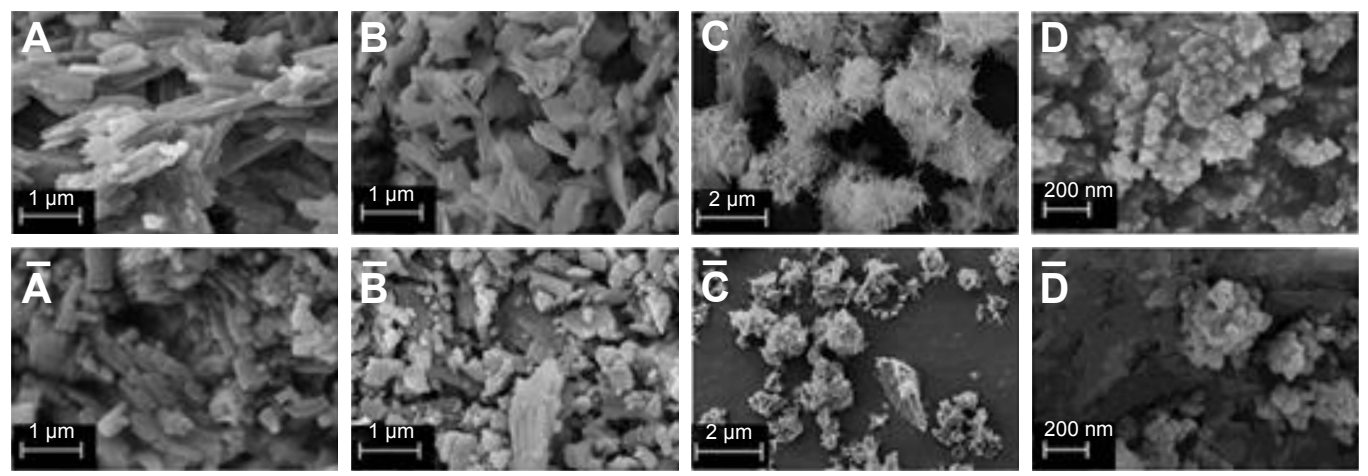

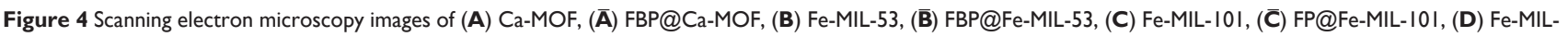

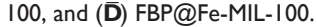

Abbreviations: FBP, flurbiprofen; MOF, metal organic framework. 
Table I Textural properties of MOFs and loading percent of FBP

\begin{tabular}{lllll}
\hline $\begin{array}{l}\text { MOF } \\
\text { characteristics }\end{array}$ & Ca-MOF & Fe-MIL-53 & Fe-MIL-I 00 & Fe-MIL-I O I \\
\hline Loading $(\%)$ & $10( \pm 1.0)$ & $20( \pm 0.8)$ & $46( \pm 3.1)$ & $37( \pm 2.3)$ \\
Surface area $\left(\mathrm{m}^{2} / \mathrm{g}\right)$ & 34.72 & 26.20 & $1,604.8 \mathrm{I}$ & 715.19 \\
Pore width $(\mathrm{nm})$ & 12.33 & 12.30 & 3.02 & 7.33 \\
Pore volume & 0.10 & 0.06 & 0.67 & 0.55 \\
$\left(\mathrm{~cm}^{3} / \mathrm{g}\right)$ & & & &
\end{tabular}

Abbreviations: FBP, flurbiprofen; MOF, metal organic framework.

desorption isotherms as shown obviously in the isotherms of Fe-MIL-100 and Fe-MIL-101.

\section{Drug loadings}

The loading percentage values of FBP on the MOFs were around $46 \%, 37 \%, 20 \%$, and $10 \%$ for Fe-MIL-100, Fe-MIL-101, Fe-MIL-53, and Ca-MOF, respectively, as shown in Table 1. The highest loading percent was on Fe-MIL-100 followed by Fe-MIL-101, which is correlated to the MOF characteristics of high surface area and pore volume. The surface areas of Fe-MIL-53 and Ca-MOF were 26.20 and $34.72 \mathrm{~m}^{2} / \mathrm{g}$, respectively, while the pore sizes were 12.3 and $12.33 \mathrm{~nm}$, respectively. The high pore size allowed for FBP molecules to be loaded but the low surface area was the reason behind low loading percent.

\section{Release profile}

The release profiles of FBP@MOFs were evaluated in $50 \mathrm{mmol}$ PBS at pH 7.4 as a serum fluid (Figure 5). The release profile demonstrated two stages for FBP-MOFs. In the first stage, the release rates for FBP@Ca-MOF, FBP@ Fe-MIL-101, and FBP@Fe-MIL-100 were around 50\% within first $2 \mathrm{~h}$, while the release rate for FBP@Fe-MIL-53 was around $65 \%$. The second stage of FBP release from

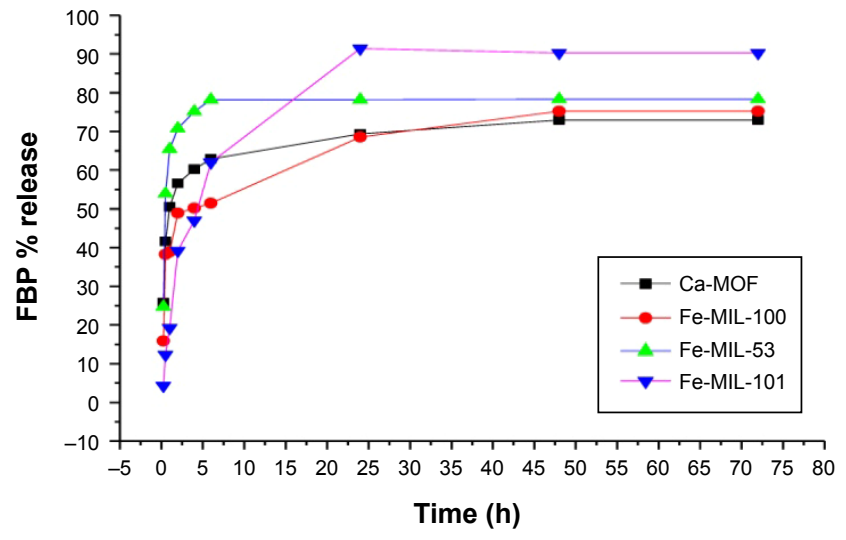

Figure 5 FBP release profiles in 50 mmol phosphate buffer solution at $\mathrm{pH} 7.4$ and $37^{\circ} \mathrm{C}$.

Abbreviations: FBP, flurbiprofen; MOF, metal organic framework.
FBP@Ca-MOF and FBP@Fe-MIL-53 was a slow stage where the FBP was released within $6 \mathrm{~h}$ and continued at stationary level for 3 days. FBP@Fe-MIL-101 exhibited the second stage within 24 h, whereas FBP@Fe-MIL-100 exhibited within $48 \mathrm{~h}$ until it reached the stationary level. The total amounts of the FBP released were $72.9 \%, 75.2 \%$, $78.3 \%$, and $90.3 \%$ for Ca-MOF, Fe-MIL-100, Fe-MIL-53, and Fe-MIL-101, respectively.

\section{Discussion}

This is the first study that employed MOFs as carrier for FBP loading. The highest FBP loading capacity with Fe-MIL-100 was because of its specific surface area $\left(1,604.81 \mathrm{~m}^{2} / \mathrm{g}\right)$ and large pore volume $\left(0.67 \mathrm{~cm}^{3} / \mathrm{g}\right)$. The lowest loading capacity with $\mathrm{Ca}-\mathrm{MOF}$ is correlated to the low surface area $\left(34.71 \mathrm{~m}^{2} / \mathrm{g}\right)$ and low pore volume $\left(0.10 \mathrm{~cm}^{3} / \mathrm{g}\right)$. The average pore size of $\mathrm{Ca}-\mathrm{MOF}$ was the highest among the employed MOFs (12.33 nm), which can open the access for FBP molecules to be encapsulated inside the pores; however, loading capacity was limited because of the small BET surface area, low pore volume and the ink-bottle pore effect. ${ }^{28}$

There are no studies or observations reporting Ca-MOF as drug delivery systems to demonstrate a comparison with these results. Although the surface area of Fe-MIL-53 was $26.2 \mathrm{~m}^{2} / \mathrm{g}$, it had a pore size of $12.3 \mathrm{~nm}$, which is enough to host the FBP molecule and achieve around 20\% loading capacity. Having a creditable surface area, pore size, and volume were the reasons behind the relative high loading capacity of Fe-MIL-101 as shown in Table 1. Horcajada et al found that both Cr-MIL-53 and Fe-MIL-53 solids adsorb around $20 \%$ (wt) of ibuprofen, ${ }^{29}$ and that there was $35 \%$ loading percent of ibuprofen with MIL-100. ${ }^{30}$ Although the FBP molecule, which is employed in this study, is larger than ibuprofen molecule, the Fe-MIL-53 loading capacity $(20 \%)$ was similar to that of Horcajada et als finding, while a significant improvement of Fe-MIL-100 loading capacity (46\%) was found. Pore size and pore volume of the MOFs alongside MOFs' surface area are the main factors that determine the manner and the percentage of the FBP loading and release with MOFs as shown in Figure S3. Large pore sizes enable different pharmaceuticals to be encapsulated. ${ }^{31}$ The drugs of poor aqueous solubility have an affinity for encapsulating with hydrophobic pores in MOFs such as the MIL family. ${ }^{2}$ Horcajada and co-workers found unprecedented amount of adsorbed ibuprofen ( 1.4 g/g MIL-101), which was due to large surface area $\left(5,510 \mathrm{~m}^{2} \mathrm{~g}^{-1}\right) .{ }^{16}$ The result of FBP loading capacity with Fe-MIL-101 was different from ibuprofen because of the large size of FBP molecule 
in comparison to the ibuprofen molecule and due to the differences in the surface area and pore size and volume of the Fe-MIL-101.

The FBP release from the FBP@MOFs showed two stages. This is related to the FBP location within the pores of MOFs, pore size, and kind of host-guest interaction. When the filled pores with FBP were located close to the surface of MOFs, FBP molecules release concerned only the weakly bonded molecules. The first stage was a fast stage of release (within $2 \mathrm{~h}$ ), which can be regarded as zero-order kinetics because of drug concentration independence. The kinetics of FBP release from FBP@MOFs are empirically adjusted with regression factors of $0.99,0.85,0.77$, and 0.78 for FBP@Fe-MIL-101, FBP@Fe-MIL-100, FBP@ Ca-MOF, and FBP@Fe-MIL-53, respectively, as shown in Figure S4. It can be defined to be a burst effect because of the porous structure and high solubility of FBP in PBS at $\mathrm{pH} 7.4$ relating to ionization due to the acidic nature of FBP (pKa 4.6). The second stage of release can be defined as an erosion process due to the slow collapse of the MOFs in alkaline media. Frameworks collapsing in PBS are due to replacement of the carboxylate linkers by phosphate groups in the PBS solution and/or formation of iron oxide rendering less favourable formation of stable metal carboxylate bonds at $\mathrm{pH}$ values above the $\mathrm{pKa}$ of carboxylic functions $(\sim 4-5) .{ }^{32}$ Cunha et al found a rapid release of caffeine from MIL-100 and 53 within $6 \mathrm{~h}$ in a medium of PBS at $\mathrm{pH} 7.4 .^{32}$ The collapsing or degradation of MOFs in the body will produce materials such as iron oxide ${ }^{33}$ or organic linkers in MOFs. ${ }^{30}$

The total percent release of FBP@Fe-MIL-100 and 53 and Ca-MOF did not reach $>90 \%$. However, the final drug fraction, probably located inside some micropores, therefore, needs more time to be completely released as a consequence of the pore diameter and cation-anion and $\pi-\pi$ interactions.

\section{Conclusion}

MOFs have maintained their integrity during the loading process. The MOFs have a variety of loading capacities. Fe-MIL-100 and 101 were the best MOFs as drug delivery systems for FBP. The overall release profile was slow for FBP to be formulated as a controlled-release drug. The MOFs are a promising material for FBP to be a carrier.

\section{Acknowledgments}

We acknowledge the School of Pharmacy, Curtin University, for providing access to their laboratories and employing different instruments and materials. The authors acknowledge the use of Curtin University's Microscopy and Microanalysis facilities, whose instrumentation has been partially funded by University, State, and Commonwealth Government.

\section{Disclosure}

The authors report no conflicts of interest in this work.

\section{References}

1. Vallet-Regí M, Balas F, Arcos D. Mesoporous materials for drug delivery. Angewandte Chemie International Edition. 2007;46(40):7548-7558.

2. Huxford RC, Della Rocca J, Lin W. Metal-organic frameworks as potential drug carriers. Current Opinion in Chemical Biology. 2010;14(2): 262-268.

3. Rosi NL, Eddaoudi M, Kim J, O'Keeffe M, Yaghi OM. Advances in the chemistry of metal-organic frameworks. Cryst Eng Comm. 2002;4(68): 401-404.

4. Meek ST, Greathouse JA, Allendorf MD. Metal-organic frameworks: a rapidly growing class of versatile nanoporous materials. Adv Mater. 2011;23(2):249-267.

5. Valtchev V, Tosheva L. Porous nanosized particles: preparation, properties, and applications. Chemical Reviews. 2013;113(8):6734-6760.

6. Liu R, Li D, He B, et al. Anti-tumor drug delivery of $\mathrm{pH}$-sensitive poly(ethylene glycol)-poly(L-histidine-)-poly(L-lactide) nanoparticles. Journal of Controlled Release. 2011;152(1):49-56.

7. Riehemann K, Schneider SW, Luger TA, Godin B, Ferrari M, Fuchs H. Nanomedicine - challenge and perspectives. Angewandte Chemie International Edition. 2009;48(5):872-897.

8. Zhang J, Wang L-Q, Wang H, Tu K. Micellization phenomena of amphiphilic block copolymers based on methoxy poly (ethylene glycol) and either crystalline or amorphous poly (caprolactone-b-lactide). Biomacromolecules. 2006;7(9):2492-2500.

9. Cheng Y, Wang J, Rao T, He X, Xu T. Pharmaceutical applications of dendrimers: promising nanocarriers for drug delivery. Frontiers in Bioscience: a Journal and Virtual Library. 2007;13:1447-1471.

10. Freiberg S, Zhu XX. Polymer microspheres for controlled drug release. Int J Pharm. 2004;282(1-2):1-18.

11. Sun C-Y, Qin C, Wang X-L, Su Z-M. Metal-organic frameworks as potential drug delivery systems. Expert Opinion on Drug Delivery. 2013; 10(1):89-101.

12. Hinks NJ, McKinlay AC, Xiao B, Wheatley PS, Morris RE. Metal organic frameworks as NO delivery materials for biological applications. Microporous and Mesoporous Materials. 2010;129(3): 330-334.

13. Keskin S, Kizılel S. Biomedical applications of metal organic frameworks. Industrial \& Engineering Chemistry Research. 2011;50(4): 1799-1812.

14. Férey G. Hybrid porous solids: past, present, future. Chemical Society Reviews. 2008;37(1):191-214.

15. Mizutani M, Maejima N, Jitsukawa K, Masuda H, Einaga H. An infinite chiral single-helical structure formed in $\mathrm{Cu}$ (II)-L-/D-glutamic acid system. Inorganica Chimica Acta. 1998;283(1):105-110.

16. Horcajada P, Serre C, Vallet-Regí M, Sebban M, Taulelle F, Férey G. Metal-organic frameworks as efficient materials for drug delivery. Angewandte chemie. 2006;118(36):6120-6124.

17. Horcajada P, Chalati T, Serre C, et al. Porous metal-organic-framework nanoscale carriers as a potential platform for drug delivery and imaging. Nature Materials. 2010;9(2):172-178.

18. Bernini MC, Fairen-JimenezD, Pasinetti M, Ramirez-Pastor AJ, Snurr RQ. Screening of bio-compatible metal-organic frameworks as potential drug carriers using Monte Carlo simulations. Journal of Materials Chemistry B. 2014;2(7):766-774.

19. Horcajada P, Chalati T, Serre C, et al. Porous metal-organic-framework nanoscale carriers as a potential platform for drug delivery and imaging. Nature Materials. 2010;9(2):172. 
20. Chalati T, Horcajada P, Couvreur P, et al. Porous metal organic framework nanoparticles to address the challenges related to busulfan encapsulation. Nanomedicine. 2011;6(10):1683-1695.

21. Sumida K, Hu M, Furukawa S, Kitagawa S. Structuralization of Ca2+-Based Metal-Organic Frameworks Prepared via Coordination Replication of Calcium Carbonate. Inorganic Chemistry. 2016;55(7): 3700-3705.

22. Anderson B, Conradi R. Predictive relationships in the water solubility of salts of a nonsteroidal anti-inflammatory drug. Journal of Pharmaceutical Sciences. 1985;74(8):815-820.

23. Begum M, Sudhakar M, Abbulu K. Flurbiprofen-loaded stealth liposomes: studies on the development, characterization, pharmacokinetics, and biodistribution. Journal of Young Pharmacists. 2012;4(4): 209-219.

24. Mokhtar M, Sammour OA, Hammad MA, Megrab NA. Effect of some formulation parameters on flurbiprofen encapsulation and release rates of niosomes prepared from proniosomes. International Journal of Pharmaceutics. 2008;361(1):104-111.

25. Bezverkhyy I, Popova E, Geoffroy N, Herbst F, Bellat J-P. Preparation of magnetic composites of MIL-53 (Fe) or MIL-100 (Fe) via partial transformation of their framework into $\gamma$-Fe $2 \mathrm{O} 3$. Journal of Materials Chemistry A. 2016;4(21):8141-8148.
26. Maksimchuk NV, Kovalenko KA, Fedin VP, Kholdeeva OA. Cyclohexane selective oxidation over metal-organic frameworks of MIL-101 family: superior catalytic activity and selectivity. Chemical Communications. 2012;48(54):6812-6814.

27. Jiang J, Yaghi OM. Brønsted acidity in metal-organic frameworks. Chemical Reviews. 2015;115(14):6966-6997.

28. Thommes M, Cychosz KA. Physical adsorption characterization of nanoporous materials: progress and challenges. Adsorption. 2014;2(20): 233-250.

29. Horcajada P, Serre C, Maurin G, et al. Flexible porous metal-organic frameworks for a controlled drug delivery. J Am Chem Soc. 2008;130(21): 6774-6780.

30. Horcajada P, Gref R, Baati T, et al. Metal-organic frameworks in biomedicine. Chemical Reviews. 2011;112(2):1232-1268.

31. Morris RE, Wheatley PS. Gas storage in nanoporous materials. Angewandte Chemie International Edition. 2008;47(27):4966-4981.

32. Cunha D, Ben Yahia M, Hall S, et al. Rationale of drug encapsulation and release from biocompatible porous metal-organic frameworks. Chemistry of Materials. 2013;25(14):2767-2776.

33. Naqvi S, Samim M, Abdin M, et al. Concentration-dependent toxicity of iron oxide nanoparticles mediated by increased oxidative stress. International Journal of Nanomedicine. 2010;5:983. 


\section{Supplementary materials \\ NMR spectroscopy}

Nuclear magnetic resonance (NMR) spectroscopy is extremely helpful in investigating the interactions between the framework and adsorbed species. Solid-state NMR experiments were performed on pure metal organic frameworks (MOFs), loaded MOFs, and pure flurbiprofen (FBP). Compared to the spectrum of pure drug, loaded MOFs show the resonance of methyl group of FBP molecule to be assigned on the $1.2 \mathrm{ppm}$ in all spectra as shown in Figure S1.

\section{$\mathrm{N}_{2}$ adsorption-desorption analysis}

$\mathrm{N}_{2}$ adsorption-desorption isotherms were obtained and analyzed to determine the surface area, pore size, and pore volume of each MOF separately. Based on the $\mathrm{N}_{2}$ adsorption isotherms, the Brunauer-Emmett-Teller surface area of all MOFs was assessed. The pore volume obtained from the desorption isotherm at P/Po 0.99 (Figure S2A-D) and the pore-size distributions derived from the $\mathrm{N}_{2}$ isotherms by the Barrett-JoynerHalenda method gave the average pore size of all MOFs.

\section{The FBP release profiles}

The release profiles of FBP@MOFs demonstrated two stages. The first stage represented by fast release profile and exhibited within 2 h. The kinetics of FBP release from FBP@MOFs are empirically adjusted with regression factors of 0.99 , 0.85, 0.77, and 0.78 for FBP@MIL-101, FBP@MIL-100, FBP@Ca-MOF, and FBP@MIL-53, respectively, as shown in Figure S3. These regression factors indicate zero-order kinetics behavior.
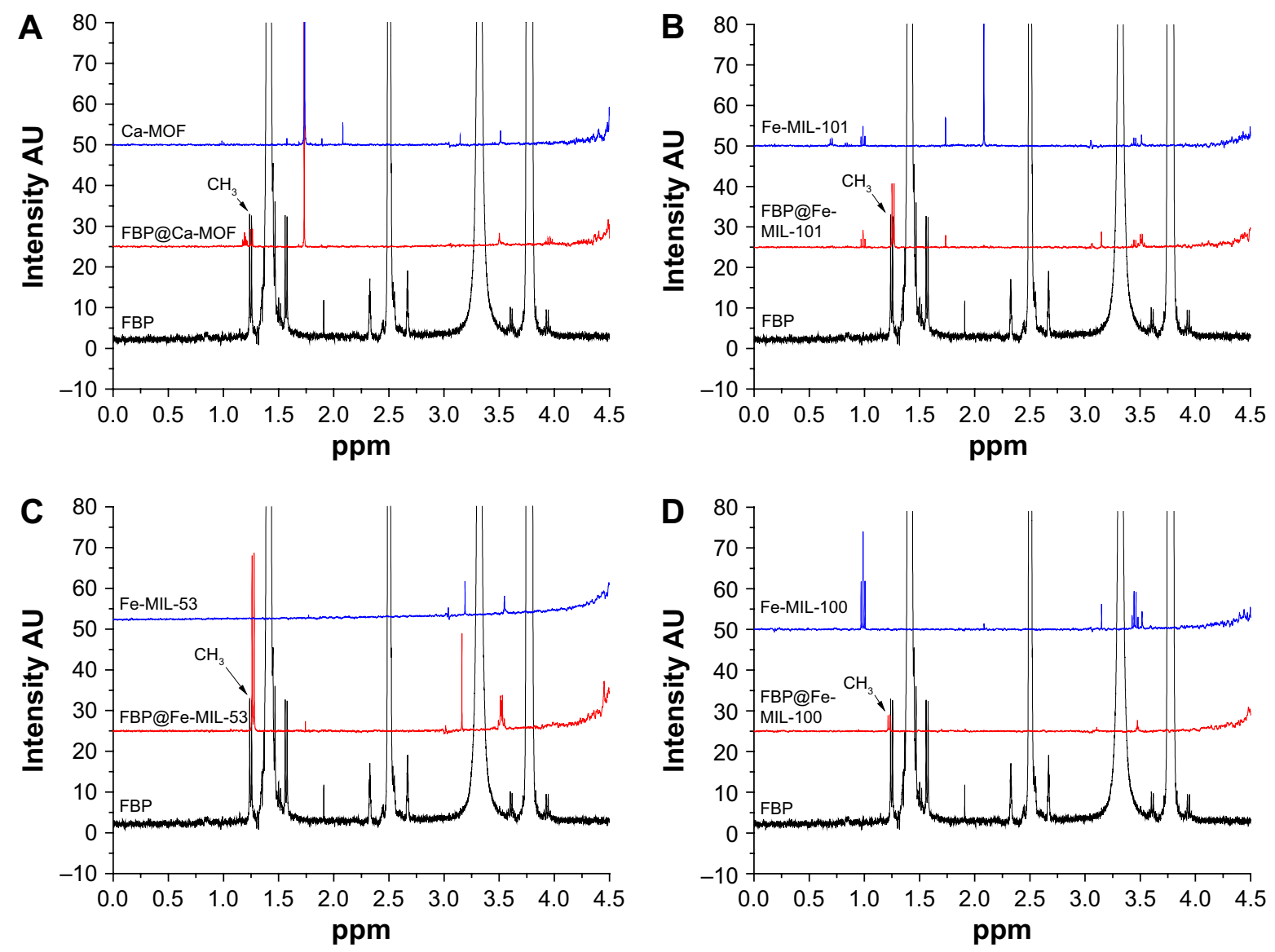

Figure SI Nuclear magnetic resonance spectrum of Ca-MOF (A), Fe-MIL-I0I (B), Fe-MIL-53 (C), and Fe-MIL-I00 (D).

Abbreviations: FBP, flurbiprofen; MOF, metal organic framework. 
A

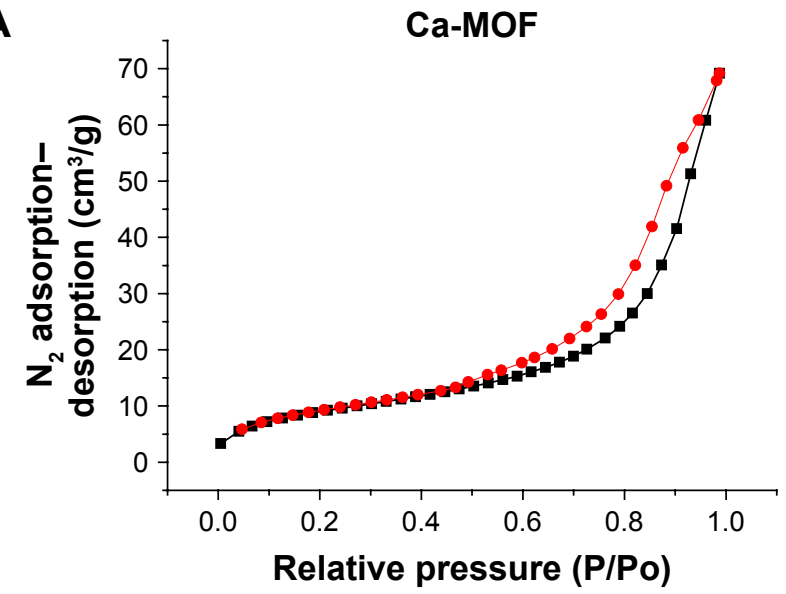

C

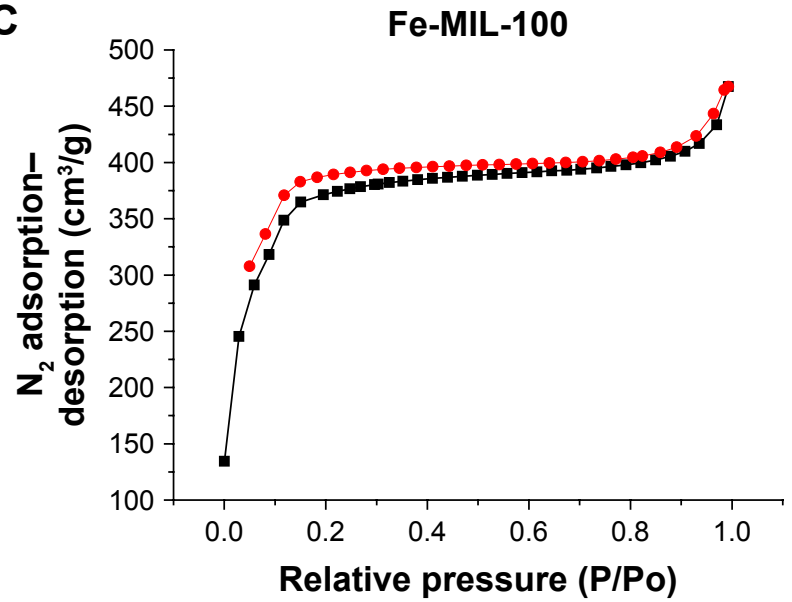

B

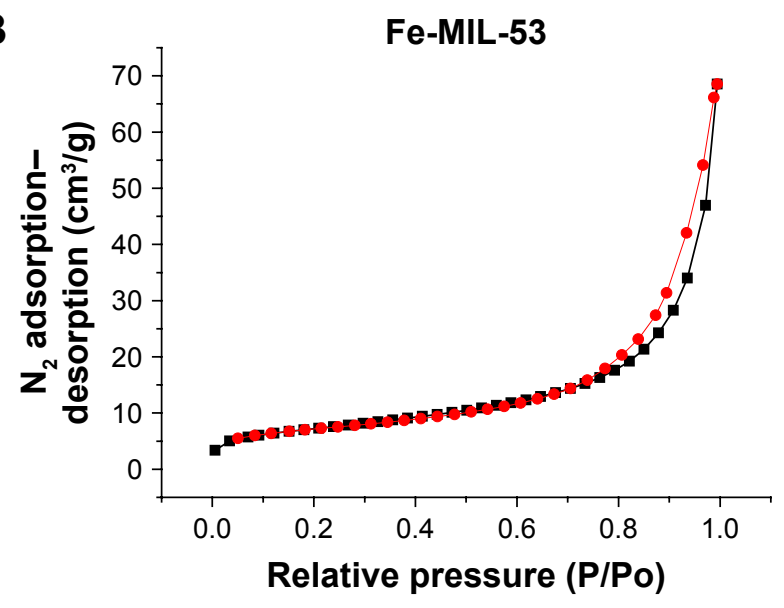

D

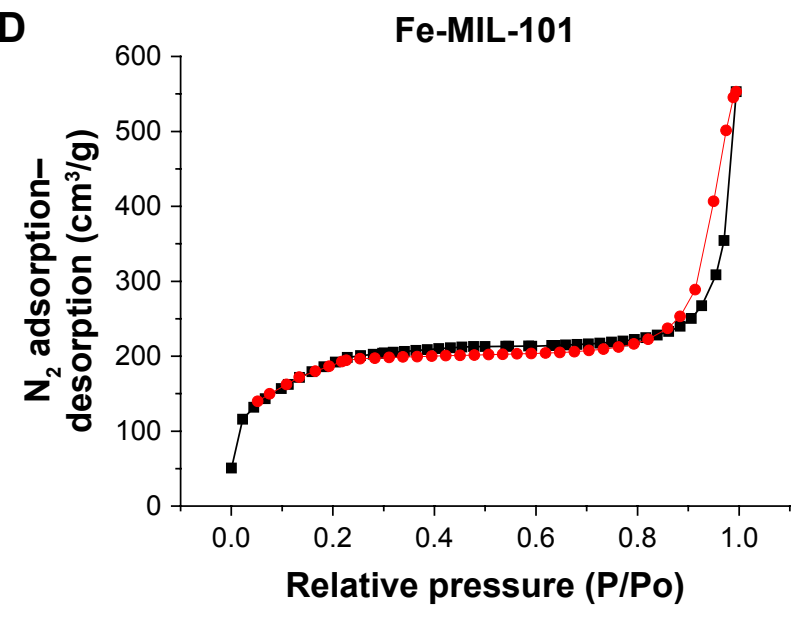

Figure S2 $\mathrm{N}_{2}$ adsorption-desorption behavior of Ca-MOF (A), Fe-MIL-53 (B), Fe-MIL-I00 (C), and Fe-MIL-I0I (D). Abbreviation: MOF, metal organic framework.

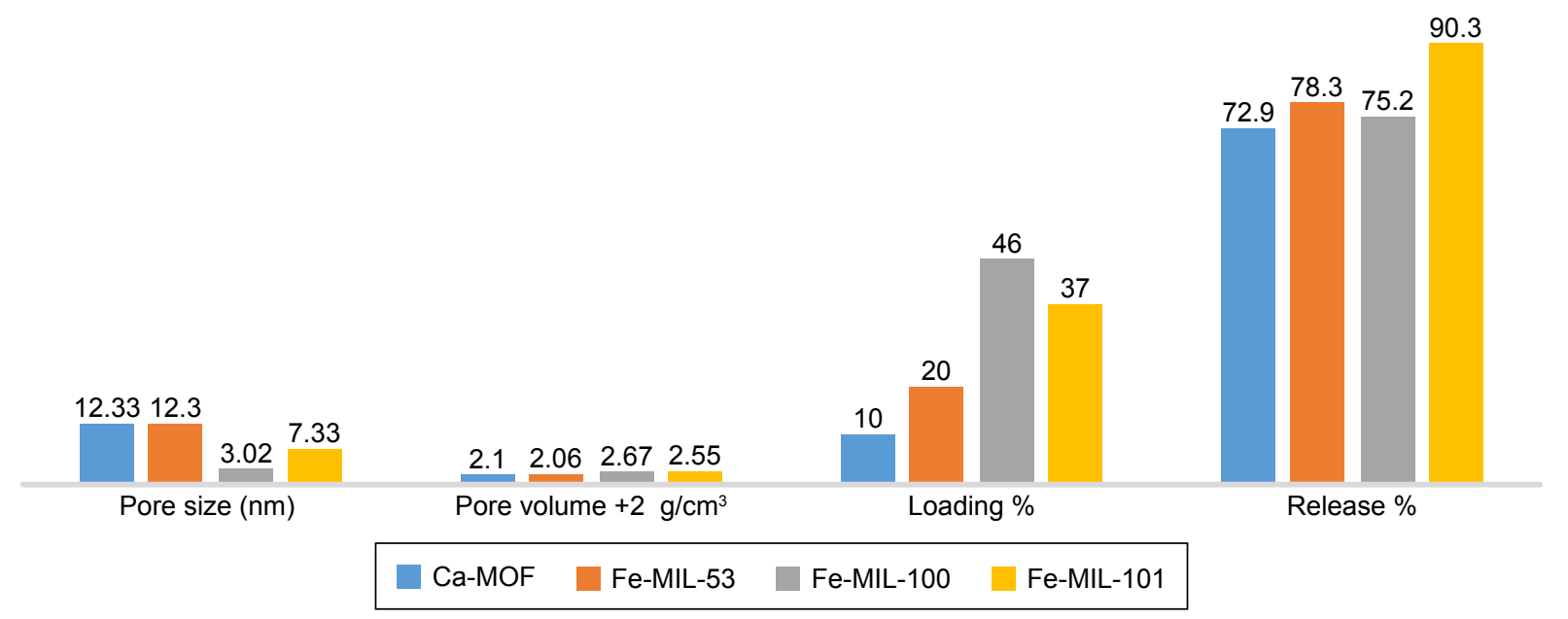

Figure S3 Relationship of pore size and volume of MOFs with the flurbiprofen loading and release percentages. Abbreviation: MOFs, metal organic frameworks. 


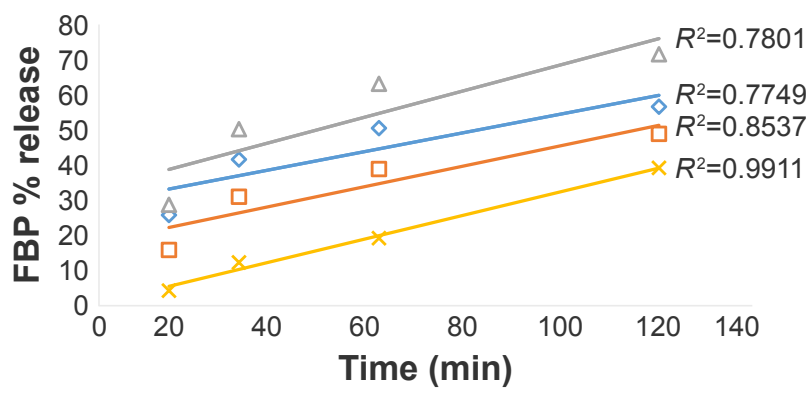

Figure S4 Kinetic order of first stage of FBP release profiles ( $\triangle$ FBP@Fe-MIL-53, $\diamond$ FBP@Ca-MOF, $\square$ FBP@Fe-MIL-I00 and X FBP@Fe-MIL-10I). Abbreviations: FBP, flurbiprofen; MOF, metal organic framework.

\section{Publish your work in this journal}

Drug Design, Development and Therapy is an international, peerreviewed open-access journal that spans the spectrum of drug design and development through to clinical applications. Clinical outcomes, patient safety, and programs for the development and effective, safe, and sustained use of medicines are the features of the journal, which has also been accepted for indexing on PubMed Central. The manuscript management system is completely online and includes a very quick and fair peer-review system, which is all easy to use. Visit http://www.dovepress.com/testimonials.php to read real quotes from published authors.

Submit your manuscript here: http://www.dovepress.com/drug-design-development-and-therapy-journal 